\title{
L'Attention
}

\section{Pour une approche phénoménologique de l'attention conjointe}

\section{Étienne Bimbenet}

\section{(2) OpenEdition}

1 Journals

Édition électronique

URL : http://journals.openedition.org/alter/1577

DOI : 10.4000/alter.1577

ISSN : 2558-7927

Éditeur :

Association ALTER, Archives Husserl (CNRS-UMR 8547)

\section{Édition imprimée}

Date de publication : 1 octobre 2010

Pagination : $93-110$

ISBN : 2-9522374-6-8

ISSN : $1249-8947$

\section{Référence électronique}

Étienne Bimbenet, «Pour une approche phénoménologique de l'attention conjointe », Alter [En ligne], 18 | 2010, mis en ligne le 01 juin 2020, consulté le 27 juin 2020. URL : http://journals.openedition.org/ alter/1577 ; DOI : https://doi.org/10.4000/alter.1577 


\title{
POUR UNE APPROCHE PHÉNOMÉNOLOGIQUE DE L'ATTENTION CONJOINTE
}

\author{
Par Étienne Bimbenet
}

En son appellation précise, «l'attention conjointe » (joint attention) est un thème de réflexion nouveau pour la philosophie. Elle fait son apparition en 19751, mais il faut attendre les années 1990 pour la voir intéresser, aussi bien les psychologues de l'enfant, que les éthologues, qu'enfin les philosophes, essentiellement du côté de ce qu'il est convenu d'appeler la «philosophie de l'esprit » anglo-saxonne (philosophy of mind ${ }^{2}$ ). Les uns et les autres reconnaissent en elle un seuil, quand ce n'est pas une frontière, séparant clairement deux types de comportements, dans l'ordre de l'ontogenèse comme dans celui de la phylogenèse. De là le succès théorique de la notion. L'attention conjointe fait événement, dans l'histoire de la vie, et pose du coup des questions majeures, qui polarisent une grande partie des discussions : faut-il voir en elle un marqueur humain fondamental ? ou bien fait-elle son apparition en amont des comportements humains ? et si tel est le cas, où la situera-t-on dans l'échelle des comportements animaux?

Sous son apparence immédiate, l'attention conjointe se définit comme le fait de partager l'attention d'autrui. Nous sommes alors deux (ou plus) à regarder un même objet («l'objet cible»). On parle de «triangle référentiel », ou encore de relation «triadique » à la chose, par opposition à une relation simplement «dyadique». Avant six mois l'enfant se rapporte soit aux choses soit aux vivants, qu'il distingue fort bien ; mais il les distingue si bien, justement, qu'il n'établit aucun lien de l'un à l'autre : « L'enfant applique sa capacité attentionnelle à deux domaines parfaitement séparés : le domaine

1. Cf. l'article pionnier de M. Scaife et J.S. Bruner, « The capacity for joint visual attention in the infant ", Nature, 253, 1975, p. 265-266, repris dans Le Développement de l'enfant. Savoir faire, savoir dire, trad. M. Deleau, PUF, 1983, p. 251-254.

2. Cf. l'ouvrage collectif paru en 1995 aux Etats-Unis, et témoignant de la richesse de cette première réflexion, C. Moore et P.J. Dunham (éd.), Joint Attention. Its Origine and Role in Development, Hillsdale, New Jersey and Hove, Lawrence Erlbaum Associates, 1995. 


\section{L'attention}

des échanges sociaux et le domaine des objets physiques [...] Par exemple, un enfant peut faire de grands efforts pour attraper un objet qui est hors de sa portée mais près de sa mère, sans même regarder cette dernière ${ }^{3}$. » Entre six et neuf mois se met en place la possibilité de coordonner les deux types d'attention ${ }^{4}$; l'attention conjointe désigne alors un ensemble de compétences diverses, mais qui ont toutes en commun « $d$ 'incorporer » dans un contexte social la relation à la chose ou au monde. On ne saurait pourtant s'en tenir à une définition strictement " géométrique » de la notion. Certes $c^{\prime}$ est bien la capacité de détecter la direction du regard de l'autre qui la première alerta les chercheurs. Mais cette capacité, partagée par bien des animaux notamment dans la compréhension que l'autre «me regarde » et qu'il y a là une menace ou au contraire un signal affiliatif, cette capacité ne dit pas l'essentiel de l'attention conjointe ${ }^{5}$. Celle-ci ne consiste pas seulement à regarder ce que l'autre regarde, mais en outre à savoir que l'autre regarde la même chose, bref : à avoir conscience d'un voir en commun. L'attention conjointe est une attention "mutuellement manifeste ${ }^{6}$ », qui consiste pour l'enfant à vérifier que l'autre regarde bien la même chose que lui (regard de contrôle), à «déclarer » la chose à l'adresse d'autrui, en la lui présentant directement (geste de montrer) ou en l'indiquant du doigt (pointage), à s'engager dans des épisodes d'interaction sociale autour d'un objet (engagement conjoint), à interroger l'attitude de l'autre pour savoir de quoi il retourne concernant l'objet (référence sociale), à commenter soi-même l'objet à l'adresse de l'autre, expressivement ou verbalement (commentaire), etc. On peut ainsi plaider pour une définition pleinement " psychologique » (et non simplement géométrique) de la notion: "L'attention conjointe est fondamentalement un phénomène social ou socio-cognitif : deux individus savent qu'ils font attention à quelque chose en commun ${ }^{7}$. »

3. L. Camaioni, «The Development of intentional communication. A re-analysis », in New Perspectives in early communication development, éd. J. Nadel et L. Camaioni, London, New York, Routledge, 1993, p. 84.

4. Ibid. Même constat chez H.R. Schaffer, The Child's Entry into a Social World, London, Academic Press, 1984, p. 79 : «Alors qu'une grande partie des six premiers mois était occupée par l'attention mutuelle qui reliait l'enfant à ses parents, désormais l'attention se transforme, incorporant des traits de l'environnement à l'interaction. "

5. Cf. S. Baron-Cohen, « The Eye Direction Detector (EDD) and the Shared Attention Mechanism (SAM) : two Cases for Evolutionary Psychology », in C. Moore et P.J. Dunham (éd.), Joint Attention. Its Origine and Role in Development, op. cit., p. 46-47.

6. N. Eilan, «Joint attention, Communication, and Mind », in N. Eilan, C. Hoerl, T. McCormack and J. Roessler (éd.), Joint Attention: Communication and other minds. Issues in Philosophy and Psychology, , Oxford, Clarendon Press, 2005, p. 5.

7. M. Tomasello, «Joint Attention as Social Cognition », in C. Moore et P.J. Dunham (éd.), Joint Attention. Its Origine and Role in Development, op. cit., p. 106. 


\section{Une perspective socio-cognitive}

Dans la définition qu'en donne Michael Tomasello - l'une des plus fameuses, et qui est aussi l'une des plus systématisantes -, l'attention conjointe se présente comme «la capacité d'organismes individuels à comprendre que leurs congénères sont comme eux, qu'ils ont une vie intentionnelle et mentale semblable à la leur ${ }^{8}$ ». Elle conjugue alors deux dimensions distinctes : premièrement, la capacité chez le tout jeune enfant (dès les premières semaines de développement) de comprendre qu'autrui est « comme lui » ; et deuxièmement, acquise plus tardivement, la conscience d'être un agent intentionnel (en un sens pratique), c'est-à-dire capable de viser des buts à travers différents moyens. Sous le premier aspect Tomasello s'en remet aux découvertes d'A. Meltzoff concernant «l'imitation néonatale ${ }^{9} »$. Constatée très tôt chez le nourrisson, une telle imitation, essentiellement faciale (ouverture de la bouche, protrusion de la langue, etc.), témoignerait d'un sens inné de l'intersubjectivité, l'enfant comprenant dès la naissance qu'autrui est «comme lui » (théorie du like me). Comme Meltzoff et Gopnik, Tomasello pense qu'on tient là une caractéristique distinctive de l'espèce humaine ${ }^{10}$. Et comme eux il pense que ce «nativisme de l'état initial ${ }^{11}$ " produit l'essentiel de ses effets plus tard, le "like me » faisant office de cadre d'accueil cognitif pour la connaissance ultérieure d'autrui, un cadre au départ relativement vide, et appelé à se remplir progressivement. On touche ici au second versant de l'attention conjointe. Vers neuf mois l'enfant se découvre comme un agent intentionnel (self agency), capable de projeter des buts qu'il sait distinguer des moyens qui y mènent. L'apparition de cette compétence instrumentale s'atteste de manière autonome, dans la relation de l'enfant aux objets qu'il manipule ${ }^{12}$; mais cette connaissance nouvelle de soi se projette alors en autrui, par le biais analogique du like me: «On peut donc faire l'hypothèse que lorsque l'enfant parvient à comprendre ses propres actions intentionnelles, il peut alors

8. M. Tomasello, Aux Origines de la cognition humaine, trad. Y. Bonin, Paris, Retz, 2004, p. 11.

9. Cf. A. Meltzoff, «La théorie du "like me" précurseur de la compréhension sociale chez le bébé : imitation, intention et intersubjectivité", in Imiter pour découvrir l'humain. Psychologie, neurobiologie, robotique et philosophie de l'esprit, éd. J. Nadel et J. Decéty, Paris, PUF (« Psychologie et science de la pensée »), 2002, p. 33-57.

10. M. Tomasello, Aux Origines de la cognition humaine, op. cit., p. 71 ; A. Meltzoff et A. Gopnik, "The role of imitation in understanding persons and developing theory of mind", in Understanding Other Minds : Perspectives from Autism, éd. S. Baron-Cohen, H. Tager-Flusberg et D.J. Cohen, New York, Oxford University Press, 1993, p. 335-366.

11.A. Meltzoff, « La théorie du "like me" précurseur de la compréhension sociale chez le bébé : imitation, intention et intersubjectivité ", art. cit., p. 34.

12. M. Tomasello, Aux Origines de la cognition humaine, op. cit., p. 72-73. Sous ce second aspect (instrumental, et non plus social) de la question, Tomasello fait référence aux analyses classiques de Piaget, in La Naissance de l'intelligence chez l'enfant, Neuchâtel, Paris, Delachaux et Niestlé, 1936. 


\section{L'attention}

utiliser le point de vue du «comme moi » pour comprendre de la même manière le comportement $\mathrm{d}^{\prime}$ autrui ${ }^{13}$.» $\mathrm{C}^{\prime}$ est parce que l'enfant a une conscience nouvelle de lui-même comme visant des buts par l'entremise de moyens distincts, qu'il peut, par projection ou simulation, comprendre autrui comme étant lui aussi un agent inentionnel. L'attention conjointe résulte ainsi d'une identification précoce à autrui, "perfectionnée » vers neuf mois à travers le raisonnement instrumental. Le premier trait est supposé spécifiquement humain, à la différence du deuxième, que nous partageons avec d'autres espèces. Les chimpanzés, par exemple, sont capables de viser un objectif et de varier leurs moyens en conséquence; mais faute de s'identifier à leurs congénères, ils "gardent pour eux» cette conscience instrumentale, agie plutôt que réfléchie à distance de $\mathrm{soi}^{14}$.

Comme on peut le voir, l'attention conjointe se conçoit chez Tomasello sur un mode essentiellement pratique. Ce qui est partagé avec autrui (par simulation analogique), ce sont des intentions de faire. Mais il n'y a là rien de restrictif. Ainsi conçue l'attention conjointe s'actualise bien sûr dans des interactions, construites autour d'un objet manipulé en commun; mais elle concerne également la perception, dès lors que celle-ci n'est pas seulement passive, mais attentive, et que se partagent dès lors les intentions de voir et d'apercevoir le même aspect du spectacle. Et il n'est pas jusqu'au langage qui ne puisse se concevoir également sur le mode d'un partage pragmatique des intentions de communication: "La compréhension d'une intention communicative est une expression particulière de la compréhension de l'intention : je dois comprendre l'intention d'autrui de s'adresser à mon état intentionnel ${ }^{15} »$. De proche en proche $c^{\prime}$ est toute la vie de conscience que l'attention conjointe permet de redéfinir à nouveaux frais, comme partage des intentions. De fait "l'attention», comme "l'intention» (pratique), sont des termes assez neutres et incolores pour pouvoir se faire les «plus petits dénominateurs communs ${ }^{16}$ ", et comme la matière première, de la conscience. On comprend alors l'extension maximale que Tomasello peut accorder à l'attention conjointe dans la vie de conscience. La notion revêt chez lui une importance considérable, élevée à la hauteur d'un critère hominisant fondamental (et au fond le seul véritable critère). Or il y a à cela une raison essentielle, qui regarde du côté des conditions empiriques de l'anthropogenèse.

Six millions d'années seulement nous séparent des grands singes; ce qui correspond à peu près au fameux $1 \%$ de différence distinguant nos équipements génétiques. De la même manière 250000 ans nous séparent des grands singes en ce qui concerne plus précisément nos compétences cognitives; c'est là encore très peu, si l'on regarde l'ampleur des

13. M. Tomasello, Aux Origines de la cognition humaine, op. cit., p. 72.

14. Ibid., p. 76.

15. Ibid., p. 98.

16. Cf. R.P. Hobson, «What puts the Jointness into Joint Attention ? », in N. Eilan, C. Hoerl, T.

McCormack and J. Roessler (éd.), Joint Attention : Communication and other minds..., op. cit., p. 186. 
transformations qui président, voilà un quart de million d'années, à l'apparition de l'homme moderne : fabrication non seulement d'outils mais de techniques industrielles particulièrement sophistiquées, invention du langage conventionnel, complexification sociale sans précédent. La temporalité biologique, celle de l'évolution naturelle, ne saurait à elle seule expliquer de telles transformations, en si peu de temps. Il faut donc compter avec une autre temporalité, historique ou culturelle : «Il n'existe qu'un seul mécanisme biologique connu capable de provoquer de tels changements dans le comportement et la cognition, dans un laps de temps aussi court (que nous prenions en compte les six millions d'années, ou seulement deux, ou même le seul dernier quart de million d'années) : la transmission sociale ou culturelle. Ce processus biologique agit sur des échelles de temps infiniment plus rapides que l'évolution organique ${ }^{17}$. » L'accumulation des ressources cognitives dans ce qu'il est convenu d'appeler une "niche culturelle» permet seule de comprendre la transformation si rapide et si spectaculaire d'un comportement. L'attention conjointe apparaît alors, sinon comme le «magic bullet ${ }^{18}$ » de l'anthropogenèse, du moins comme la condition nécessaire de l'intense travail socio-culturel qu amena un jour une population d'Homo à entamer une trajectoire biologique sans précédent. La possibilité de s'identifier au congénère comme agent intentionnel fonde en effet la possibilité de la coopération, de l'imitation, de l'enseignement, mais aussi du langage, conçu comme manipulation interactive de symboles communs, bref de tout ce qui chez un vivant permet de «mettre en commun les ressources cognitives ${ }^{19}$ ». En ceci l'attention conjointe répond exactement au problème chronologique posé par l'hominisation. Une différence biologique minimale, car unique - en l'occurrence, la capacité d'identification au congénère - suffit à produire les plus grands effets du côté non plus du biologique mais de la sociogenèse et de l'accumulation culturelle. "Les êtres humains détiennent une capacité héritée à vivre de manière culturelle $[\ldots]$ Cette infime différence biologique entre les humains et leurs plus proches parents primates a eu, et continue d'avoir les conséquences cognitives les plus considérables ${ }^{20}$. » Ainsi une espèce qui se contenterait d'être ingénieuse, mais qui ne saurait pas capitaliser ses propres inventions en les communiquant de congénère à congénère, n'aurait aucune possibilité de faire évoluer, autrement que par voie de sélection naturelle passive, ses comportements. "L'évolution culturelle cumulative n'a pas seulement besoin d'inventions créatives : il faut également, et c'est tout aussi important, qu'une transmission sociale bien précise joue ce rôle de cliquet

17. M. Tomasello, Aux Origines de la cognition humaine, op. cit., p. 9.

18. Ibid., p. 16: "Magic bullet" : allusion à la théorie de la commission Warren, selon laquelle une seule arme aurait été impliquée dans l'assassinat du président Kennedy. Une seule balle, «magique », aurait parcouru une trajectoire inexplicable dans le corps de la victime... »

19. Ibid., p. 11.

20. Ibid., p. 54 . 


\section{L'attention}

empêchant que $l^{\prime}$ on ne reparte en arrière ${ }^{21}$. » $C^{\prime}$ est dire qu'il revient à l'attention conjointe, ni plus ni moins que d'accomplir le dépassement naturel du naturel, en direction d'une temporalité historique et d'un univers de culture.

Si l'attention conjointe peut prétendre annexer, en extension, tous les comportements humains, $c^{\prime}$ est manifestement qu'elle touche à l'essentiel de ces comportements, et qu'elle semble rendre compte de ce qui les définit en propre. Or on peut se demander si Tomasello se donne vraiment tous les moyens de placer l'attention conjointe au centre de la vie de conscience. Il en suggère l'idée, mais à propos du langage seulement, si bien qu'il rate finalement la teneur véritablement «eidétique» de la question. Il note à plusieurs reprises, par exemple, qu'un symbole linguistique n'est pas seulement collectivement partagé, mais qu'il est en outre et pour la même raison sous-tendu par une perspective particulière: on signifie toujours un objet ou un état de fait selon tel aspect, mais qu'un autre pourrait dire sous tel autre aspect ; la même chose que j'offre peut être appelée une "rose", mais également une "fleur", ou une "preuve d'amour", etc ${ }^{22}$. Tomasello ne fait que constater ce perspectivisme propre au langage, mais il ne semble pas l'imputer à l'espace pluriel ouvert par l'attention conjointe en tant que telle; encore moins en tire-t-il un enseignement concernant la conscience en général. Et pourtant l'attention conjointe pourrait bien, en ouvrant l'espace commun d'une multiplicité perspective, donner l'occasion d'une véritable redéfinition en profondeur de la conscience. Ce que Tomasello dit du seul langage concerne peut-être la conscience comme telle; encore faut-il le montrer, en s'appuyant sur des intuitions eidétiquement efficaces. Or nous pensons que la phénoménologie a un rôle crucial à jouer dans une telle démonstration.

Il se trouve en effet que la "philosophie de l'esprit» (philosophy of mind), et plus spécifiquement la «théorie de l'esprit»(theory of mind), représentent, depuis la fin des années 1980, le cadre d'accueil théorique, manifestement obligé, de la notion ${ }^{23}$. La conception de Tomasello rentre de plain-pied dans ce cadre interprétatif. L'héritage biologique humain est un héritage cognitif, au sens fort de ce terme: il me donne une connaissance innée de moi-même et par là d'autrui, il me fait connaître autrui comme un autre moi-même (like me); et cette connaissance de l'autre, au départ toute formelle et rudimentaire (crude), s'affine avec le temps: à neuf mois la conscience nouvelle que j'ai de moi, non plus seulement comme être animé, mais comme sujet de décisions possibles (voulant telle chose, et capable pour y parvenir d'engager plusieurs stratégies comportementales possibles), cette conscience se projette ipso facto en autrui et modifie la perception que

21. Ibid., p. 10.

22. Ibid., p. 102.

23. En témoigne exemplairement le recueil collectif publié en 1995 par C. Moore et P.J. Dunham, Joint Attention. Its Origin and Role in Development, op. cit. 
j'en ai. Nous sommes devenus l'un et l'autre des êtres volontaires, décidés, deux agents intentionnels se connaissant comme tels l'un l'autre. L'attention conjointe, comme capacité de détecter les intentions de l'autre, apparaît ainsi comme une étape cruciale dans l'accomplissement de ce qui, pour la théorie de l'esprit, représente le fin mot de toute interaction sociale : l'accès à des états mentaux qui ne sont pas les miens. Pour bien des philosophes de cette mouvance, et au-delà de la définition strictement pratique que Tomasello donne de l'état mental, une telle capacité est anthropologiquement définitoire: "L'attribution d'états mentaux est aux hommes ce que l'écholocation est à la chauve-souris ${ }^{24}$. Elle trouve sa consécration vers l'âge de quatre ans dans le fameux test des croyances fausses: à cet âge l'enfant sait distinguer entre sa propre vue de la situation et celle, fausse, qu'un autre peut en avoir. Il peut savoir par exemple que le chocolat se trouve dans le placard bleu et comprendre en même temps que la marionnette Maxi, mal informée des déplacements du chocolat, puisse à tort le chercher dans le placard vert. À trois ans en revanche aucun enfant ne sait accomplir ce "transfert inattendu » (unexpected transfer) et se mettre à la place de Maxi, pour voir la situation du point de vue (erroné) qui est le $\operatorname{sien}^{25}$. Que cette capacité d'attribuer à l'autre des croyances qui ne sont pas les miennes soit mise au compte d'un apprentissage individuel, comme on voit chez Gopnik, Meltzoff ou Tomasello26, ou qu'elle soit imputée à la maturation d'un module cognitif implémenté au niveau de l'espèce, comme on voit chez Leslie ou Baron-Cohen ${ }^{27}$, dans tous les cas l'attention conjointe joue le rôle d'une étape décisive, dans la connaissance des états mentaux de l'autre. On reconnaît là ce qu'on appelle communément la "théorie de l'esprit» : celle-ci se reconnaît en effet au fait que le rapport à autrui s'y définit chaque fois comme un problème - le problème posé par son esprit. Que ce soit sur le mode théorique d'un déchiffrement (mind-reading) des

24. S. Baron-Cohen citant D. Sperber, in Mindblindness : an essay on autism and theory of mind, Cambridge, MIT Press, 1995, p. 3-4. Cf. également M. Davies et T. Stone, Folk Psychology. The Theory of Mind Debate, Oxford, Blackwell, 1995.

25. J. Perner, Understanding the Representational Mind, Cambridge (Mass.), London (UK), MIT Press, 1991, p. 179.

26. C'est la théorie (que nous évoquions plus haut) du «nativisme de l'état initial », qui ne confie à l'héritage biologique que la connaissance liminaire, toute formelle, qu'autrui est " comme moi »; tout le reste est affaire de maturation individuelle et d'apprentissage culturel. Cf. A. Meltzoff, « La théorie du "like me" précurseur de la compréhension sociale chez le bébé : imitation, intention et intersubjectivité », art. cit.

27. Chez S. Baron-Cohen ou A. Leslie, toute l'architecture de la théorie de l'esprit doit se concevoir comme un ensemble de modules cognitifs enchassés, et implémentés au niveau de l'espèce. Il faut ainsi que s'actualisent EDD (eye direction detector), SAM (shared attention mechanism) et ToDD (theory of mind mechanism) pour mener progressivement l'enfant vers une intelligence "proustienne » de l'autre. Cf. A. Leslie, « Pretence and Representation : the origins of "Theory of mind" ", Psychological Review, 94, 1987, p. 412-426 ; S. Baron-Cohen, "The Eye Direction Detector (EDD) and the Shared Attention Mechanism (SAM) : two cases for Evolutionary Psychology », art. cit. 


\section{L'attention}

comportements d'autrui (theory theory), ou sur le mode pratique d'une simulation immédiate (simulation theory), dans les deux cas ma connaissance d'autrui n'est rien qui va de soi, au regard de la connaissance au contraire native que j'ai de mes propres états mentaux. Or on aperçoit de part et d'autre deux des préjugés qui saturent, de manière hypercartésienne, le cognitivisme caractéristique de la théorie de l'esprit. Un préjugé mentaliste, d'abord, voudrait nous faire croire que l'esprit est un domaine intérieur, un ensemble de représentations accessibles à un seul, et que donc l'autre «se cache " nécessairement derrière son corps. Je ne peux accéder à l'autre vie que par analogie avec ma propre vie - en la devinant à travers les indices qu'en donne son comportement, ou en adoptant son point de vue en première personne, bref en enjambant, sur un mode théorique ou simulé, toute la distance qui m'en sépare. Le deuxième préjugé représente moins une illusion qu'une abstraction, ou un rétrécissement du regard : dans la construction de Tomasello, comme plus généralement dans la théorie de l'esprit, l'essentiel porte sur le rapport à autrui. En sa désignation même, «l'attention conjointe » s'offre à nous prioritairement comme un phénomène social ; elle fait événement dans la seule connaissance que l'enfant (ou le vivant) peut avoir de ses congénères, et ce au détriment de la structure globale de son rapport au monde.

Or on peut voir les choses autrement. En sa rigueur descriptive, la phénoménologie dénonce comme illusoires ou abstraits, d'un côté le mythe d'un esprit insulaire, primitivement intime à lui-même, et de l'autre l'idée qu'on ne sort de chez soi que pour aller s'enquérir d'autrui. Sur le premier point, on répondra à la suite de Scheler ou Merleau-Ponty qu'autrui n'est pas nécessairement moins bien connu que nous-même, y compris, et même surtout, chez le tout jeune enfant; et sur le deuxième point, on fera valoir une analyse intentionnelle pour examiner la manière dont l'attention conjointe transforme, au-delà du seul rapport à autrui, le rapport au monde lui-même. Revenons tour à tour sur ces deux aspects de l'analyse; il y va d'une tout autre définition du phénomène que celle qu'accrédite, d'une manière faussement naturelle, la Théorie de l'esprit.

\section{L'attention séparante}

L'approche phénoménologique des faits humains n'est jamais sans passé. Lui échoit souvent, en provenance de la psychologie, une certaine rigueur descriptive, qu'en réalité elle n'ignore jamais. En ce qui concerne l'attention conjointe, il revient en l'occurrence à une certaine psychologie du développement de nous orienter, contre le mentalisme des philosophes, vers les choses mêmes. Des auteurs comme C. Trevarthen ou P. Hobson comprennent la communication préverbale de l'enfant en des termes largement désintellectualisés au regard de l'approche cognitive. Ici il n'y a plus à partir de soi et de la connaissance de ses propres états mentaux pour 
expliquer, sur un mode théorique ou simulationniste, le comportement de l'autre. Le rapport de l'enfant à autrui est (1) non pas cognitif mais affectif, et (2) il ne donne aucune précédence au moi sur autrui. Nous sommes plongés ici dans l'élément brûlant des premiers liens, en un lieu où l'autre ne peut plus apparaître, sauf par pétition de principe, comme un être à connaître et à déduire depuis soi. Nous voici alors rendus à l'opposé de l'intellectualisme et du mentalisme qui sous-tendent la théorie de l'esprit. D'une part l'enfant se rapporte émotionnellement à l'autre, il n'est plus question de déchiffrer ou d'expliquer son comportement; la connaissance est un mode d'être qui vient trop tard au regard des « qualités viscérales " (blood-and-guts qualities ${ }^{28}$ ) qui composent la vie du premier âge. Mais d'autre part l'idée d'une intériorité transparente à elle-même et dont tout découlerait, l'idée que l'autre serait "lui aussi » une intériorité qu'il y aurait à déchiffrer ou pénétrer, une telle idée ne résiste pas à une description rigoureuse de l'intersubjectivité. L'enfant saisit l'autre immédiatement, à même ses attitudes et ses expressions. Celles-ci peuvent être faciales, vocales ou même gestuelles, l'autre y sera toujours présent en personne et non à déduire depuis soi sur la base d'indices corporels. "Percevoir un sourire comme sourire (pour prendre l'exemple le plus simple), c'est lui répondre affectivement (with feeling), d'une manière telle qu'à travers ce sourire se donne l'état émotionnel de l'autre ${ }^{29}$. » Dans les «protoconversations »du nourrisson avec l'adulte, il n'y a pas la connaissance d'un «moi » puis la déduction d'un «il», mais un «moi » et un «toi » émotionnellement résonnants, mimétiquement accordés, et donc contemporains l'un de $l^{\prime}$ autre ${ }^{30}$. Il n'y a pas à « aller » vers l'autre, à enjamber la distance qui sépare les deux corps, à percer le mur d'une intériorité étrangère : car l'autre est là, donné à même son corps, aussi immédiatement que je suis donné à moimême. Comment comprendre alors, dans un cadre théorique ainsi renouvelé, l'attention conjointe ? Il n'est plus question ici de mieux connaître autrui, ou d'en percer le mystère, puisque celui-ci est dès le départ visible en son être même. Simplement dans l'attention conjointe la relation dyadique, en face-àface, se transforme au profit d'une relation triadique, qui intègre l'objet dans la relation à autrui. De «l'intersubjectivité primaire » à l'intersubjectivité secondaire $^{31}$ » le passage est continu. S'il est vrai qu'une attitude ou une expression est toujours intentionnellement dirigée vers un état du monde, il suffit que l'enfant comprenne plus finement ces attitudes et leur sens propre,

28. R.P. Hobson, «What puts Jointness into Joint Attention? », in N. Eilan, C. Hoerl, T. McCormack and J. Roessler (éd.), Joint Attention: Communication and other minds. Issues in Philosophy and Psychology, op. cit., p. 187.

29. Ibid., p. 190.

30. Cf. J. Heal, "Joint Attention and Understanding the Mind", art. cit., in N. Eilan, C. Hoerl, T. McCormack et J. Roessler (éd.), Joint Attention: Communication and other minds. Issues in Philosophy and Psychology, op. cit., p. 41.

31. Cf. C. Trevarthen, "Descriptive analysis of infant communicative behaviour", in Studies in Mother-Infant Interaction, éd. H.R. Schaffer, London, Academic Press, 1977. 


\section{L'attention}

pour que la relation à l'autre se complique d'une ouverture au monde. "Tout ce dont on a besoin dans ce cas, au-delà du savoir partagé de l'espace, $c^{\prime}$ est de savoir que l'autre est en train de regarder et d'expérimenter quelque chose dans le monde visuel ${ }^{32} »$. De même que le rapport à autrui, l'attention conjointe est ici à peine un problème : on glisse sans heurt de la perception $\mathrm{d}^{\prime}$ autrui à la compréhension de ce qu'il fait, d'une saisie émotionnelle de ses expressions à une lecture du sens intentionnel de ses comportements.

Le grand mérite de cette "théorie de l'intersubjectivité33 » (par opposition à la théorie de l'esprit), c'est d'assurer sur une base phénoménologiquement fiable le rapport à autrui chez l'enfant, mais plus généralement en toute vie humaine. Ici il ne peut plus être question, sauf reconstruction «adultocentrique ", de penser la vie de l'enfant sur un mode théorique et mentaliste, comme c'est le cas avec le like me. Car une telle projection analogique du moi dans l'autre donne en réalité beaucoup trop à l'autre dans la vie de l'enfant. Non que l'enfant dans les premiers temps ne soit happé par les autres, en particulier par ses parents. Il l'est au contraire à corps perdu ; « entre lui et ceux de son entourage il n'y a que des traits d'union ${ }^{34}$ ». Mais justement : un tel rapport lui interdit de se rapporter à un autre que soi. Si l'autre est partout dans la vie de l'enfant, il n'est en même temps nulle part, parce que donné paradoxalement sans son altérité. L'intersubjectivité telle que la vit l'enfant ne va jamais jusqu'à la position de l'autre comme autre, telle que la postule le like me. Cela regarde la donation d'autrui en général: il est tout simplement faux de dire, dans les termes de la théorie de l'esprit, que l'autre est une vie cachée derrière ce que j'en aperçois. Le mentalisme est phénoménologiquement contredit par l'expérience que nous faisons des comportements vivants. En ceux-ci l'autre nous est donné en personne, à même ses gestes, ses attitudes, ses expressions faciales, ses intonations. La donation charnelle d'autrui condamne le raisonnement par analogie à venir toujours trop tard, comme Scheler l'avait stigmatisé. Pour simuler la joie de celui qui me fait face, il faut déjà saisir que c'est de la joie qui s'exprime sur son visage et que c'est elle que j'ai à répliquer. "L'existence d'expériences internes, de sentiments intimes, nous est révélée dans et par les phénomènes d'expression, c'est-à-dire que nous en acquérons la connaissance, non à la suite d'un raisonnement, mais d'une façon immédiate, au sens d'une « perception » originaire et primitive. Nous percevons la pudeur de quelqu'un

32. J. Bruner, «From Joint Attention to Meeting of Minds: an Introduction », in Joint Attention. Its Origin and Role in Development, op. cit., p. 7.

33. Nous appellerons ainsi la ligne interprétative développée à la suite de C. Trevarthen et P. Hobson par des auteurs comme J. Heal ou J. Roessler, in N. Eilan, C. Hoerl, T. McCormack et J. Roessler (éd.), Joint Attention: Communication and other minds. Issues in Philosophy and Psychology, op. cit. À cet égard, ce dernier ouvrage (publié en 2005) répond efficacement à l'ouvrage de C. Moore et P.J. Dunham (publié en 1995), dans lequel au contraire prévalait largement l'interprétation cognitiviste du phénomène.

34. H. Wallon, De l'Acte à la Pensée. Essai de psychologie comparée, Paris, Flammarion, 1942, p. 101. 
dans sa rougeur, la joie dans le rire ${ }^{35}$. $\gg$ Le raisonnement par analogie est illégitime non seulement parce qu'il raisonne, mais surtout parce que, posant que le psychisme est ce qui ne peut $\mathrm{m}^{\prime}$ apparaître que dans le secret d'une intériorité, il se condamne à ne jamais rencontrer autrui. C'est pourquoi il ne peut fonctionner qu'en présupposant ce qui est à poser : il faut avoir perçu le sens d'une expression pour pouvoir la revivre soimême $^{36}$. Ainsi nous sommes d'ores et déjà d'intelligence avec l'autre, et non confrontés à une énigme. L'enfant n'est pas plus familier de lui-même et du sentiment qu'il a de lui-même, que d'autrui. L'un se perçoit aussi immédiatement que l'autre, selon une théorie de "l'intersubjectivité innée » dont les arguments derniers, regardant vers la donation expressive d'autrui, sont phénoménologiques plutôt que biologiques : «Il est juste faux de dire que les états mentaux humains ne sont pas observables. Nous pouvons «détecter» les états mentaux des autres instantanément dans leurs expressions, sans entraînement [...] Un sourire est heureux, comme l'est un pas rapide et léger ; les larmes sont tristes, comme l'est une marche lente et traînante, ou un regard abattu; et les émotions exprimées par quelqu'un peuvent entraîner une mimique instantanément compatissante chez un autre $^{37}$ ». Instantanément lisible en ses expressions, l'autre est donné dans une proximité telle que ce ne peut pas être comme autre qu'il se présente.

Tout se passe donc comme si l'enfant ne connaissait pas autrui, pour en être justement trop proche ; comme s'il le ratait, par trop de proximité. On peut vivre avec l'autre sans le reconnaître comme autre, tel pourrait être, nous semble-t-il, l'enseignement dispensé par la psychologie de la prime enfance. La découverte, récente, des neurones miroirs, semble venir conforter cette conclusion. Découverts dans le cortex prémoteur ventral du macaque (aire F5), puis chez l'homme au niveau de l'aire de Broca, ces neurones ont ceci de remarquable qu'ils codent aussi bien l'exécution d'un acte finalisé (prendre un objet avec la main, par exemple), que l'observation de ce même acte, accompli cette fois par un congénère. Percevoir ce que fait quelqu'un (le voir prendre un verre, agripper un bâton), c'est refaire avec lui cet acte même, d'une manière dite " couverte $^{38}$ ». On a pu parler ainsi de «systèmes résonnants »; d'où l'espoir d'une redéfinition en profondeur de

35. Cf. M. Scheler, Nature et formes de la sympathie, trad. M. Lefebvre, Paris, Payot, 2003, p. 56.

36. Cf. M. Merleau-Ponty, La Structure du comportement, op. cit., p. 169: «Toute théorie de la projection, qu'elle soit empiriste ou intellectualiste, suppose ce qu'elle voudrait expliquer, puisque nous ne pourrions projeter nos sentiments dans le comportement visible d'un animal, si quelque chose dans ce comportement même ne nous suggérait l'inférence ».

37. C. Trevarthen, "The self born in intersubjectivity: the psychology of an infant communicationg ", in U. Neisser (éd.), The Self perceived. Ecological and interpersonal sources of selfknowledge, New York, Cambridge University Press, 1993, p. 122 et 151.

38. Cf. G. Rizzolatti et G. Sinagaglia, Les Neurones miroirs, trad. fr. M. Raiola, Paris, Odile Jacob, 2008. 


\section{L'attention}

notre rapport à autrui, en particulier depuis le concept de simulation ${ }^{39}$. Or l'interprétation simulationniste des neurones miroirs est loin d'aller de soi. Elle rencontre en particulier le même type d'objections que celles avancées naguère par Scheler à l'encontre du transfert analogique ${ }^{40}$. À sa faveur s'accrédite en effet implicitement l'idée d'une précession de moi sur autrui, ou d'une projection du moi dans le toi. Or une interprétation plus économique des neurones miroirs consisterait simplement à dire que les deux corps ont en commun un lexique d'actes moteurs, qui n'est pas davantage le mien que celui de l'autre. Je comprends l'autre geste sans avoir à me mettre à la place d'autrui, parce que ce geste, avec la situation qui est la sienne (son but, ses points d'application) est entre nous et à nous deux, comme tout ce que nous percevons. « Lorsque je jette un œil par la fenêtre et que je vois un homme traverser la route, ma propre perception de l'environnement suffit à me faire comprendre que son point de vue s'ouvre sur le même environnement que le mien, et la plupart du temps je n'ai pas besoin d'en savoir plus. Je n'ai pas à me mettre à sa place pour savoir qu'il voit la route selon un angle de vue qui diffère du mien. ${ }^{41}$ »Ceci, c'est la description phénoménologique de notre expérience qui le constate; mais, comme ajoute S. Gallagher, il n'y a pas de raison de faire fonctionner différemment les neurones miroirs: "Le système miroir n'est pas un commencement de simulation; il participe d'une perception directe de ce que l'autre est en train de faire ${ }^{42}$. » La simulation est un transfert de moi à autrui qui donne trop à la séparation du moi et du toi, là où la résonance plaide au contraire en faveur de leur indifférenciation face à la neutralité des buts poursuivis. Une interprétation "perceptive" des neurones miroirs rejoint ainsi directement la définition «perceptive » du rapport à autrui chez le tout jeune enfant: dans les deux cas l'autre est directement lisible à partir du sens intentionnel de ses comportements. Il n'y a pas à pénétrer une intériorité mais à comprendre quel but est poursuivi, dans quelle situation et dans quel contexte.

39. V. Gallese et A. Goldman, « Mirror Neurons and the Simulation Theory of Mind-Reading », Trends in Neuroscience, 2, 1998, p. 493-501.

40. Cf. G. Csibra, "Mirror neurons and action observation. Is simulation involved ?», 2005, version imprimable in http://www.interdisciplines.org/mirror, p. 3: "Si vraiment la simulation est impliquée dans le fonctionnement des neurones miroirs, le singe «simule » parce qu'il a compris une action, plutôt qu'il comprend une action parce qu'il l'a simulée ».

41. S. Gallagher, "Logical and phenomenological arguments against simulation theory", in D. Hutto et M. Ratcliffe (éd.), Minding our Practice : Folk Psychology Re-assessed, Springer Publishers, p. 6 .

42. Ibid., p. 10. Cf. également M. Jeannerod et E. Pacherie, "Agency, simulation, and selfidentification", Mind and Language, 19, 2, p. 139: «Perception et action sont intimement intégrées l'une à l'autre, et lorsque nous percevons visuellement des actions, nous avons l'air d'être immédiatement sensibles aux propriétés caractéristiques d'un comportement intentionnel » (nous soulignons). 
Ainsi dans l'ordre des relations à autrui la communication précède la séparation. Nous naissons sous le signe du lien, et non de l'isolement; comme dit C. Trevarthen, " une subtile et immédiate conscience d'être avec l'autre vient en premier ${ }^{43}$ " . De cette première thèse, on tirera la conclusion suivante : l'attention conjointe, à partir des neuf mois, ne saurait consister à « conjoindre » deux consciences originellement séparées. Les termes de jonction, conjonction, union ou réunion, tout ce lexique pousse à une interprétation spontanée du phénomène qui n'est pas forcément la meilleure. En réalité l'attention conjointe sépare, plutôt qu'elle ne conjoint. Il faudrait entendre comme un jeu de mot plein de sens l'expression d' «attention partagée », le partage signifiant scission autant que réunion. Les épisodes $d$ 'interaction conjointe réunissent bien deux individus autour d'un faire en commun, mais justement deux "individus", sommés d'apparier deux points de vue au moins spatialement incompatibles et donc concurrents sur la même chose. C'est exactement ce qui rend cruciale, chez Tomasello, la définition du congénère comme agent intentionnel. Un être qui désormais apparaît comme poursuivant ses propres objectifs, et non plus seulement comme source de mouvements et d'effets prévisibles, un être qui fait des choix et qui prend des décisions, un tel être ne s'anticipe plus aussi facilement qu'un simple organisme. Il a davantage que «le pouvoir de faire en sorte que, globalement, les choses adviennent d'une certaine manière ${ }^{44}$ » : il produit un enchaînement réglé de gestes dont il est seul à détenir la clé, pour en avoir choisi lui-même les modalités. Soit le spectacle d'un homme qui prend une bouteille d'eau, se sert un verre, le porte à ses lèvres, et le boit. C'est une chose de saisir en chaque étape la signification émotionnelle du geste, par une forme de résonance motrice ou d'intelligence charnelle en quoi se définit l'intersubjectivité primaire; c'en est une autre, bien différente, de saisir la logique pratique qui préside à l'enchaînement des gestes et qui fait des premiers les moyens d'arriver au dernier. Car dans ce dernier cas je ne saisis plus un ensemble de buts ponctuels, immédiatement communicables d'un corps à l'autre (empoigner une bouteille, boire), mais une succession de gestes dont je ne peux savoir avant la fin quel était leur sens. La finalité de chacun des gestes n'est plus immédiatement appropriable car elle s'attend au terme seulement de la séquence motrice. Le fait que je ne puisse saisir avant la dernière étape la signification des différents moments impose une forme de délai qui fait de moi le spectateur d'une action dont je ne détiens pas la clé. Ici il est bien évident que l'autre fait quelque chose que je ne fais pas. Nous sommes passés en régime d'attention conjointe, et ce non pas parce que nous faisons attention ensemble à la même action (boire un verre), mais parce que j'assiste désormais à une suite de gestes ordonnée à une autre vue de la situation que la mienne. Dans l'attention conjointe l'enfant

43. C. Trevarthen, « The self born in intersubjectivity : the psychology of an infant communicationg », in The Self perceived. Ecological and interpersonal sources of self-knowledge, op. cit., p. 122.

44. M. Tomasello, Aux Origines de la cognition humaine, op. cit., p. 74. 


\section{L'attention}

commence à s'initier à une certaine "opacité référentielle 45 », sur un mode encore rudimentaire. C'est ainsi que la tâche de la fausse croyance à l'âge de quatre ans apparaîtra comme un progrès pour avoir, non pas rapproché l'enfant des autres (de leurs représentations intérieures), mais au contraire pour l'en avoir séparé un peu plus, en confortant chez lui le pouvoir d'imaginer des croyances opposées aux siennes. D'une manière générale, il faudrait concevoir la maturation comme l'assomption progressive, au cœur de la vie de l'enfant, de l'altérité de l'autre. Grandir n'est pas se socialiser mais au contraire desserrer l'étau des attachements primitifs, faire à l'autre sa place et continuer à le comprendre, malgré la distance.

L'attention conjointe implique une certaine déférence: elle accorde à l'autre autant qu'à soi, autrement dit ce pouvoir exorbitant donné à la vie de faire apparaître tout l'apparaissant. Supposer ce pouvoir donné à la naissance, comme le fait la théorie du like me, c'est méconnaître ce qu'il en coûte à un enfant de se relativiser, lui qui était jusqu'ici au centre. À partir des neuf mois commence à s'entrouvrir le voile d'une vie dans laquelle les autres peuvent me faire apercevoir des aspects du monde que je n'avais pas vus, ou plus brutalement encore donner tort à l'aspect que je croyais jusqu'ici le seul réel. Dans cette distance creusée entre moi et autrui, la théorie de l'esprit semble reprendre des droits. Elle dit quelque chose d'essentiel concernant non l'enfant mais l'adulte; quelque chose qui, pointant avec l'attention conjointe, est faux avant elle, mais vrai après. Dans une vie adulte l'autre est "comme moi» (like me); alors (mais alors seulement) nous sommes les uns à l'égard des autres des alter ego, capables de dévoiler chacun des aspects du monde réel ou vrai. En ceci l'attention conjointe "intellectualise" notre rapport à autrui. Mais c'est à condition d'ajouter que l'essentiel est moins dans cette intellectualisation elle-même que dans la scission et la réorganisation de l'expérience qu'elle induit. L'essentiel, c'est que pour la première fois l'enfant n'est plus seul face à son monde ; c'est ni plus ni moins que l'altérité de l'autre qui s'entraperçoit ; et celle-ci ne peut l'être que si l'enfant parvient à voir l'autre comme voyant "lui aussi " la chose, comme étant "lui aussi» au centre du monde. L'attribution à l'autre d'états mentaux épistémiques, du type perception ou croyance, introduit entre lui et moi la plus grande distance qui se puisse imaginer : celle qui sépare deux points de vue sur le monde. Voir que l'autre voit lui aussi ce que je vois, ou croire qu'il a sur un sujet sa propre croyance, $c^{\prime}$ est désormais cohabiter avec d'autres consciences. Ainsi la question n'est pas d'approfondir la connaissance d'autrui et de ses états psychiques; elle est autrement radicale. Une connaissance plus précise d'autrui et de ses états mentaux ne fait pas une conduite nouvelle, quoi qu'on veuille croire. $\mathrm{Si}$ l'attention conjointe est si novatrice, $c^{\prime}$ est qu' elle donne pour la première fois à l'enfant l'idée qu'il n'est pas le seul point de vue sur le monde ; avec elle

45. S. Baron-Cohen, La Cécité mentale. Un essai sur l'autisme et la théorie de l'esprit, trad. J. Nadel et F. Lefebvre, PUG, 1998, p. 67. 
s'annonce la nécessité de partager tout l'existant avec d'autres, qui y ont le même droit que moi.

Ainsi la part faite aux autres est-elle plus ou moins grande dans une vie d'homme. Mais nous savons maintenant qu'elle n'est pas ce dont nous partons, mais qu'elle se conquiert et s'augmente en grandissant. À chaque fois l'enfant mûrit l'altérité de l'autre, parallèlement à une certaine " flexibilité imaginative ${ }^{46}$ »; à chaque fois s'augmente le pouvoir d'être un autre ou de n'être pas réel, ce qui revient au même. Nous devenons nousmêmes en nous déprenant de nous-mêmes; nous grandissons en gonflant notre expérience de voix, de gestes et d'attitudes qui nous viennent d'ailleurs. Reste que cette distance à soi ne peut pas ne pas s'inscrire dans notre perception du monde: $c^{\prime}$ est ce second aspect, non plus social mais intentionnel, qu'il nous faut maintenant examiner.

\section{La transcendance du monde}

En réalité l'avènement de l'altérité de l'autre, dans l'attention conjointe, ne saurait laisser indemne le monde lui-même, tel que j'en fais l'expérience. Tel est, si l'on y pense, l'enseignement que nous ont délivré conjointement la phénoménologie et la psychologie du développement, concernant l'intersubjectivité en général : celle-ci, dès la plus élémentaire résonance motrice, rejoint l'autre dans ses buts et dans les objets qu'il manipule ; nous ne déchiffrons pas des états internes cachés derrière des gestes abstraits, nous percevons ces gestes avec le sens intentionnel qui est le leur. Aussi élémentaire soit ce sens (prendre un objet à pleine main, en force, ou au contraire en douceur, etc.), il est ce que nous percevons à même le comportement. C'est pourquoi le rapport à autrui chez l'enfant est sinon nécessairement, du moins potentiellement triadique. Il nous faut donc préciser les choses, du côté d'une analyse clairement intentionnelle ou situationnelle du rapport à l'autre. La question n'est pas de savoir à quel moment l'objet s'immisce dans ce rapport, puisqu'il y est inscrit au moins virtuellement dès l'origine, mais plutôt de savoir en quoi la distance progressivement mise entre soi et l'autre, et plus radialement la reconnaissance de l'autre en son altérité, finit par induire une perception nouvelle de l'objet.

De fait le monde n'est plus le même, d'être visé non plus solitairement mais à plusieurs. En comptant avec les autres dans ma vue du monde j'inscris désormais en ce dernier une multiplicité de vues possibles. L'attention conjointe n'est pas conjonction mais disjonction, elle fait de ma vue du monde un "point de vue » possible parmi d'autres; mais du coup le monde se multiplie, comme objet d'une multiplicité perspective. D'être visé à plusieurs le monde m'apparaît comme n'étant plus «ma chose », il recule

46. P. Harris, «From simulation to folk psychology: the case for development », Mind and language, 7, 1992, p. 131. 


\section{L'attention}

dans une transcendance exactement mesurée par l'altérité d'autrui. Le monde est profond : riche d'aspects inépuisables, explorable indéfiniment, parce que le perçoit un corps lui-même profond, riche de sa communication avec d'autres corps. L'essentiel du cube est dans ses faces cachées, dans cette réserve inépuisable qui propose à mon regard une chose véritablement existante. Ainsi en m'ouvrant à l'altérité de l'autre l'attention conjointe m'ouvre à celle du monde ; la distance qui me sépare d'autrui est corrélative de celle qui me sépare désormais du monde. L'intentionnalité qui advient à cet âge désigne la visée d'un objet, c'est-à-dire d'un être qui me fait face, n'étant pas réductible à la visée que j'en ai. Définie de cette manière l'intentionalité porte en elle, comme son secret natal, la présence d'autrui ou sa présence comme absent: la déprésentation de la chose visée, ce qui l'empêche de m'apparaître comme une projection mienne, tient au fait que je ne suis pas autrui, qu'il n'est pas moi, et que je dois désormais faire, dans ma visée du monde, avec ce regard venu d'ailleurs. "L'acte de la référence émerge non pas comme un acte individuel, mais social : par l'échange des choses avec l'Autre, par le fait de les toucher et de les regarder avec $l^{\prime}$ Autre ${ }^{47}$. "Selon l'équation fameuse de Werner et Kaplan, la "situation primordiale du partage ${ }^{48}$ » opère la transformation des «choses d'action » en « objets de contemplation ${ }^{49}$ ». Parce que la chose n'est plus exclusivement la mienne, parce que je la partage désormais avec d'autres, elle n'est plus exactement ce que je peux manipuler pour moi, elle est déjà bonne à contempler plutôt qu'à prendre. L'intentionnalité s'inaugure, en son sens spécifiquement humain, de cette intrusion d'autrui dans mon paysage. Tout ce qui m'entoure est virtuellement partageable, voilà ce qui brise le cercle égocentrique de l'agir et conditionne l'apparition d'objets, jetés en face de moi comme autres à moi-même.

Il semble que nous tenions là une équation spécifiquement humaine : ce serait une seule et même chose pour nous de reconnaître l'altérité d'autrui et la transcendance du monde, plus tard la réciprocité des ego et l'objectivité de l'objet. Sous son premier aspect (celui du rapport à autrui), l'attention conjointe creuse l'écart qui nous sépare des autres. Certes l'enfant commence par avoir accès, sur le mode d'une perception immédiate, à la situation commune; mais cette condition originelle doit faire, en grandissant, avec l'idée que l'autre n'est pas moi; la perception y compose désormais avec l'imagination. Un enfant est appelé à se construire dans une suite $\mathrm{d}$ 'imitations qui valent chaque fois non pas comme des répétitions mais au contraire comme l'exploration d'attitudes inédites, de postures avantageuses, de gestes qui l'augmentent. Il intégrera l'univers humain de la culture en prenant aux autres tout ce qu'il pourra leur prendre, mais en se déprenant

47. H. Werner et B. Kaplan, Symbol formation. An Organismic-Development Approach to Language and the Expression of Thought, John Wiley and Sons, Inc., New York, London, Sydney, p. 43. 48. Ibid., p. 42.

49. Ibid., p. 44. 
toujours plus de lui-même, en apprenant de la bouche et du corps d'autrui celui-là qu'il était. Ce décentrement systématique, Merleau-Ponty l'a thématisé à travers le concept de "chair », ce corps définitivement impropre, mimétiquement vagabond, empathiquement happé par tous les autres corps alentour $^{50}$. Le corps humain est ainsi fait qu'il ne s'appartient pas. C'est un corps analogique, constamment fait, défait, contrefait par ses rôles, ses simulations et ses faire-semblants. Grand lecteur de Paul Schilder 51 , Merleau-Ponty comprend l'ontogenèse humaine (en tant qu'humaine) comme l'histoire d'un corps se construisant par une altération progressive de soi, un exercice d'imagination et d'exploration. Mais on ne saurait en rester là, sauf à reproduire l'erreur de la théorie de l'esprit, qui est d'enfermer l'être humain dans sa socialité, au détriment du monde. Il se trouve précisément que la chair, chez Merleau-Ponty, commence à devenir un concept intrigant, insistant, offert pour longtemps à la méditation, lorsqu'il met en regard ce corps ubiquitaire avec une définition corrélative du monde. On passe alors d'une anthropologie de la culture (du corps socialisé et malléable à merci) à une anthropologie véritablement philosophique, capable d'apercevoir un nouveau mode d'être dans l'histoire de la vie. D'où ce second aspect de l'attention conjointe, inséparable du premier : le monde de l'homme est ce qui constitutivement lui échappe. Parce que mon corps s'échappe à lui-même, le monde se fuit en des lignes d'horizon sans fin. Il y a autant d'imaginaire dans mon rapport à moi-même (de rôles appris, d'identifications rêvées, de fantasmes enfouis) que dans la texture du monde, gonflée par tout ce que j'aurais pu voir ou qu'un autre aurait pu voir à ma place. De même que l'essentiel de mon corps appartient à autrui, de même l'essentiel de la chose vue est dans son revers invisible, dans les «faces cachées du cube». En sa profondeur inépuisable le monde est fondamentalement ce que nous voyons à plusieurs: «Le sensible est précisément ce qui, sans bouger de sa place, peut hanter plus d'un corps. [...] Tout repose sur la richesse insurpassable, sur la miraculeuse multiplication du sensible. Elle fait que les mêmes choses ont la force d'être choses pour plus d'un [...] Toute l'énigme est dans le sensible, dans cette télé-vision qui nous fait au plus privé de notre vie simultanés avec les autres et avec le monde ${ }^{52}$. » Intentionnellement décrite, la distance mise entre moi et autrui se marque dans le monde même, à travers l'avènement d'un perspectivisme ouvert. Nous donnons sur un monde centré partout ailleurs qu'en nousmêmes parce que nous avons appris, en grandissant, que nous n'étions pas seuls au monde.

50. Cf. M. Merleau-Ponty, Le Visible et l'Invisible, op. cit., p. 172-204.

51. Cf. P. Schilder, L'Image du corps. Étude des forces constructives de la psychè, trad. F. Gantheret et P. Truffert, Paris, Gallimard, 1968.

52. M. Merleau-Ponty, Signes, Paris, Gallimard, 1960, p. 22-24. 


\section{L'attention}

\section{Conclusion}

En se donnant une représentation contestable (mentaliste) et partielle (exclusivement sociale) de l'attention conjointe, la Théorie de l'esprit se condamne finalement à en rater toute l'ampleur. Faute de bien décrire notre rapport à autrui, chez l'enfant comme chez l'adulte, et faute d'apercevoir toutes les implications de la socialité humaine, elle ne restitue ni la définition exacte ni la dimension structurale (globale) du phénomène qu'elle étudie. Le regard phénoménologique fait valoir de son côté une tout autre définition de la chose. Ici l'attention conjointe sépare, plutôt qu'elle ne rapproche ; si nous commençons par le «nous » plutôt que par le «Je", alors le progrès majeur de la petite enfance consiste à apprendre qu'il y a d'autres vies que la mienne, ou d'autres vues que la mienne sur le monde. L'attention conjointe est dissociante, plutôt qu'unissante; la conscience humaine pratique l'analyse, et non la synthèse. Par ailleurs cette transformation du rapport à autrui est inséparable d'une transformation du rapport au monde. Avec l'approfondissement de la socialité, c'est un tout nouveau rapport au monde qui s'inaugure, caractéristique de notre manière humaine d'exister. Le monde est pour nous profond, indéfiniment explorable, parce que partagé avec tous les êtres vivants que je pourrais rencontrer. Nous sommes arrachés à nous-mêmes en direction du monde, parce que nous le sommes à chaque moment en direction d'autrui. C'est dire qu'au moins deux fois l'attention conjointe nous apprend qu'une conscience humaine est moins une présence à soi ou un retour à soi, le rapatriement du divers de l'expérience sous l'unité d'un concept, que préalablement une extase ou une déhiscence l'exposition de soi à d'autres que soi, comme à autre chose que soi. Nous sommes humains non par la conscience de soi (ce soi que tout vivant connaît depuis son premier souffle), mais par la capacité de connaître un autre que soi, et autre chose que soi. 